\title{
Revista Chilena de Pediatría: 90 años de historia
}

\author{
Revista Chilena de Pediatría: 90 years of story
}

\section{Luisa Schonhaut Berman ${ }^{\mathrm{a}, \mathrm{d}}$, Teresa Millan Klüsse ${ }^{\mathrm{b}, \mathrm{d}}$, Ana J. Zepeda Ortega ${ }^{\mathrm{cd}}$}

${ }^{a}$ Clínica Alemana, Facultad de Medicina, Universidad del Desarrollo, Chile

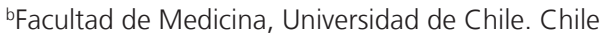

‘Escuela de Tecnología Médica, Facultad de Medicina, Universidad de Valparaíso y Centro Interdisciplinario de Investigación en Salud Territorial (CIISTE), Chile

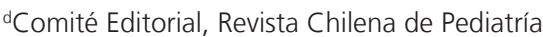

Recibido: 23 de enero de 2020 Aceptado: 29 de enero de 2020

¿Qué se sabe del tema que trata este estudio?

Revista Chilena de Pediatría ha sido publicada en forma ininterrumpida desde hace 90 años.
¿Qué aporta este estudio a lo ya conocido?

Se revisan los principales hitos de la historia de Revista Chilena de Pediatría, que la han llevado al posicionamiento en los rankings internacionales como revista de corriente principal.

\section{Resumen}

Revista Chilena de Pediatría (RCHP) se ha publicado en forma ininterrumpida desde su primera edición en enero de 1930, sorteando vaivenes políticos y económicos, innovación tecnológica y transformaciones sociales. El objetivo del presente manuscrito es homenajear y agradecer a los editores que participaron tan activamente de la publicación de RCHP y repasar los grandes hitos, que la han llevado al posicionamiento que tiene hoy como revista de corriente principal. Desde su ingreso al mundo digital hace 2 décadas, se ha registrado un importante aumento en el flujo de manuscritos recibidos, participación de autores extranjeros, citas recibidas y ascenso en los rankings internacionales. En el año 2014 RCHP fue incorporada a la National Library of Medicine (NLM) y el 2017 al Emerging Sources Citation Index (ESCI) mientras se evalúa para recibir un Factor de Impacto (FI) de parte del Journal Citation Report (JCR). Como revista representante de una sociedad científica, es fundamental mantener el equilibrio entre la sustentabilidad científica y la económica, entre la promoción de la publicación nacional y la internacionalización, entre el impacto científico requerido para continuar ascendiendo en los rankings internacionales y su rol en la educación continua, con impacto en la atención y cuidado de nuestros niños, en los distintos rincones del país.
Palabras clave:

Pediatría;

Revista;

Factor de impacto,

Publicación;

historia de la medicina

Correspondencia:

Luisa Schonhaut B.

Ischonhaut@alemana.cl 


\section{Abstract}

Revista Chilena de Pediatría (RCHP) has been published uninterruptedly since its first edition in January 1930, beyond political and economic fluctuations, technological innovation, and social transformations. The objective of this article is to pay tribute to and thank the editors who participated so actively in the publication of the RCHP and to review the major milestones, which have led it to the position it has today as a mainstream magazine. Since its entry into the digital world two decades ago, there has been a significant increase in the flow of manuscripts received, participation of foreign authors, citations received and promotion in international rankings. In 2014, the RCHP was incorporated into the National Library of Medicine (NLM) and, in 2017, into the Emerging Sources Citation Index (ESCI), while evaluating to receive an Impact Factor (IF) from the Journal Citation Report (JCR). As a representative magazine of a scientific society, it is essential to maintain a balance between scientific and economic sustainability, the promotion of national and international publication, the scientific impact need for continuing rising in international rankings, and the impact on our children>s care in the different corners of the country.

\section{Keywords:}

Pediatrics;

Journal;

Impact Factor;

Publications;

History of medicine

\section{Introducción}

En el año 1922 se fundó la Sociedad Chilena de Pediatría (SOCHIPE), siendo su primer director el Dr. Luis Calvo Mackenna. En julio de ese mismo año, Revista Médica de Chile publicaría en un número especial las actas de las sesiones preparatorias y de constitución de la SOCHIPE, además de una selección de los primeros trabajos científicos presentados en sus sesiones de la recién fundada sociedad. En septiembre de 1924 comenzó a imprimirse la primera revista, Archivos Chilenos de Pediatría, bajo la dirección de Guillermo Morales Beltramí, con la reimpresión del mismo número especial que se había publicado en la Revista Médica de Chile ${ }^{1,2}$.

En aquellos años, el contexto social, demográfico y epidemiológico del país era dramáticamente diferente al que tenemos hoy; como consecuencia de la caída de la bolsa de valores de Nueva York y la "Gran Depresión”" la economía chilena se derrumbó, incrementó la cesantía y pobreza, y las dificultades económicas de la recién nacida SOCHIPE obligaron interrumpir la publicación de Archivos Chilenos de Pediatría en el año 1927.

Afortunadamente, gracias al "espíritu de unión, sentido de solidaridad profesional, conciencia pediátrica", y la necesidad de difundir los progresos de la naciente Escuela Pediátrica Chilena, se volvió a impulsar una publicación nacional dos años después. “...un órgano de publicidad en que pudiéramos recopilar el tesoro científico de nuestra experimentación y de nuestro estudio". Con esas palabras del Dr. Arturo Baeza Goñi se dio comienzo a la Revista Chilena de Pediatría (RCHP), cuyo primer número fue impreso en enero de $1930^{4}$. El financiamiento provenía de algunas donaciones, sumado a un cobro de suscripción anual por $\$ 40$; el aporte de la SOCHIPE era escaso debido al reducido número de socios, que en los años 30 sumaban 74. A los pocos años se agregaron los avisos publicitarios, que en los 40 llegaron a costear la mitad del valor de las publicaciones; en 1940 el valor de la suscripción anual aumentaría a 80 pesos, equivalente a 3 dólares para los extranjeros. En los años 80, RCHP comenzó a recibir un subsidio de la Comisión Nacional de Investigación Científica y Tecnológica (CONICYT) que duró hasta comienzos del $2000^{1,5}$.

Desde su primera edición, hace 9 décadas, RCHP se ha publicado en forma ininterrumpida, sorteando vaivenes políticos y económicos, cambios e innovación tecnológica y transformaciones sociales ${ }^{6}$. El objetivo del presente manuscrito es homenajear y agradecer a los editores que participaron tan activamente de la publicación de RCHP y repasar los grandes hitos, que la han llevado al posicionamiento que tiene hoy como revista de corriente principal.

\section{Contexto social, demográfico, epidemiológico y perfil de publicaciones}

Entre 1930 y 2017, la población chilena se cuadruplicó, pasando de 4.287.445 a 18.419.192 de habitantes. En 1930, la mitad de la población vivía en áreas rurales (50,6\%), llegando a 11,6\% en el año 2019. Asimismo, la población de 60 años y más incrementó de 5,7\% a $16,2 \%$. Además, el porcentaje de la población de 15 años o más que no sabía leer ni escribir disminuyó de $24,4 \%$ a $3,6 \%^{7,8}$. Mientras que la esperanza de vida al nacer para ambos sexos, entre 1930 y 2019 se duplicó, de 40,6 a 80,6 años ${ }^{9,10}$.

En conjunto con lo anterior, se observó una significativa disminución de la mortalidad general, donde, de $24,1 \%$ en 1930 descendió a 6,0\% ${ }^{7,8}$. En relación con la mortalidad infantil en el año 1930, Chile se encontra- 
ban entre los países con las tasas más altas del mundo, $213 \% 0^{11}$, tasa que en menos de un siglo descendió a $7,1 \%$ o NV ${ }^{10}$. Entre 1930 y 2017 la razón de mortalidad materna disminuyó de 90 a 2,19 x 10.000 12,13 . Al respecto cabe mencionar también que la atención profesional del parto no alcanzaba al 50\% hace 90 años, la que se incrementó significativamente luego de la creación del Sistema Nacional de Salud en el año $1952^{14}$, siendo en la actualidad $99,7 \%{ }^{13}$ Otro de los indicadores que da cuenta de cómo se ha comportado la dinámica de población de nuestro país es la Tasa Global de Fecundidad, que ha disminuido de 5,5 a 1,6 hijos por mujer en edad fértil, cifra que nos ubica entre los países que están, a nivel mundial, bajo la tasa de reemplazo de su población ${ }^{15,16}$.

En síntesis; hace 90 años, nacían y morían muchos niños, había altos índices de orfandad, abandono y vagancia ${ }^{17}$. Las casas de Huérfanos o expósitos estaban repletas, siendo uno de los principales escenarios para la docencia e investigación pediátrica junto con los hospitales infantiles "Roberto del Río" y, en menor medida el "Manuel Arriarán"2,18. Los niños morían de enfermedades infecciosas y desnutrición, y se desarrollaban los primeros ensayos terapéuticos y profilácticos, temáticas que dominaban las primeras publicaciones. En el primer número de la RCHP, se publicaron los manuscritos: Distrepsias de origen psíquico; El servicio social de protección a la madre; El equilibrio ácido base en los trastornos agudos del lactante; Invaginación intestinal y Septicemia escarlatinosa con localizaciones múltiples. En los años 40 se celebró el éxito de la incorporación de tratamientos antibióticos y el impacto de las vacunas en la morbimortalidad infantil, todo documentado en RCHP por connotados investigadores $^{1}$.

En la medida que fue aumentando la sobrevida y la expectativa de vida, la morbilidad que enfrenta la población se relaciona con la transición epidemiológica y la "nueva morbilidad" pediátrica ${ }^{19}$, temas que han ido cobrando cada vez mayor espacio en RCHP y, en coincidencia con ello, se abrió a un nuevo universo de autores relacionados con la salud de los niños, más allá del enfoque biomédico. A partir del año 2000, los manuscritos que estudiaban la desnutrición y enfermedades carenciales fueron desplazados por un incrementando exponencial de publicaciones relacionadas con la obesidad, enfermedades crónicas no transmisibles, niños y adolescentes con necesidades especiales de atención en salud y salud mental.

\section{Grandes hitos de RCHP}

En sus 90 años RCHP ha tenido un total de 12 editores (tabla 1). El primero fue el Dr. Arturo Baeza
Goñi, quien continuaría en la dirección de la revista por 24 años, con un breve paréntesis en que fue reemplazado por el Dr. Gustavo García entre los años 1942 y 1944. La labor del editor fue reconocida por el Dr. Adalberto Steeger, entonces presidente de la Sociedad Chilena de Pediatría, quien, en una carta fechada con el 18 de enero de 1963, aceptaba públicamente la renuncia del entonces editor de RCHP Dr. Raúl Hernández "... con tanto acierto y esfuerzo ha servido durante 3 años. Le ha tocado actuar en épocas de profunda crisis en la vida de la Sociedad, que indudablemente ha dificultado aún más la de por sí ya pesada, contante y difícil labor del Director". Desde inicios de RCHP, el equipo editorial, trabajando estrechamente con el editor, ha cumplido un rol fundamental en el desarrollo de la revista, y son tantos los profesionales que han pasado por esta función en estas nueve décadas, que no sería posible nombrarlos a todos.

Durante esta larga historia de la revista, su portada y gráfica también ha vivido varias modificaciones. En el año 1956 se incorporó el logo con Il Bambino de L'ospedale degli Innocenti ${ }^{20}$. El año 1998 la portada se llenaba de vida con fotos de niños, hijos de los mismos pediatras y profesionales de la SOCHIPE. El año 2007, se optó por una portada más sobria desde el punto de vista de su colorido, el cual se ha mantenido hasta ahora. Esto, a su vez, disminuyó el costo asociado al diseño y la impresión.

El reglamento de las publicaciones, que comenzó a aparecer en la revista el año 1952, en un principio ocupaba apenas media página, extendiéndose en la medida que pasaban los años. En los 80 era de una carilla completa, y destacaba la exigencia de publicar los

Tabla 1. Editores Revista Chilena de Pediatría 1930 a la actualidad

\begin{tabular}{ll}
\hline Año & Editor \\
\hline $1930-1941$ & Arturo Baeza Goñi \\
$1942-1944$ & Gustavo García Álvarez \\
$1945-1953$ & Arturo Baeza Goñi \\
$1954-1955$ & Oscar Correa Bórquez \\
$1956-1957$ & Humberto Garcés Cuadra \\
$1958-1959$ & Werner Bustamante Espinoza \\
$1960-1962$ & Raúl Hernández Marchant \\
$1963-1972$ & Julio Schwarzenberg Lobeck \\
$1973-1974$ & Luis Givovich Mercier \\
$1975-1976$ & Armando Díaz Cruz \\
$1977-1980$ & Jaime Herrera Valle \\
$1981-1998$ & Carlos Toro Álvarez \\
1999 a la actualidad & Francisco Cano Schuffeneger \\
\hline
\end{tabular}


resúmenes en inglés y español; lo interesante, es que previo a ello no había uniformidad, $y$, en caso de haber resumen, este podía ser publicado en español, inglés, francés e incluso alemán. En 1986, a la página de "reglamento de publicaciones" se agregaron 5 páginas de "instrucciones a los autores" donde se especifican aspectos éticos de publicación, como la declaración del conflicto de intereses, las autorías o las publicaciones duplicadas, el respeto a la confidencialidad de la información, además de la aprobación por un comité "ad hoc" de la institución en que se realizó el estudio. La exigencia de aprobación por un comité de ética de la investigación en seres humanos se hizo presente el año 2006 y para los casos clínicos el 2017.

En la década del 60, bajo la dirección del Dr. Julio Schwarzenberg se invitó a prestigiosos profesores europeos y americanos a publicar los manuscritos en su idioma natal. En esos años, el grueso de la publicación se concentraba en la sección "revista de revistas" en que se resumía en escasas líneas manuscritos destacados de las principales publicaciones pediátricas del mundo; gran parte de estas publicaciones se obtenían por intercambio, lo que, a su vez, permitía dar a conocer RCHP a nivel internacional5; de este modo, hubo un periodo que incluso la revista Pediatrics publicaba los resúmenes en inglés de manuscritos latinoamericanos. Los títulos de gran parte de los manuscritos publicados en RCHP hasta el año 1995 pueden ser rescatados de Pubmed.

En 1963 apareció el primer sumario y se fue afinando la línea editorial, publicándose manuscritos mas estructurados, con formato IMRYD (introducción, material y método, resultados y discusión). El año 1973 se publicaron las primeras editoriales y en 1977, "con la finalidad de dar cabida a un mayor número de contribuciones" se agregaron los "contribuciones cortas" y "notas al editor". En 1990 se agregó el índice en español e inglés.

El Dr. Carlos Toro Álvarez, asumió como editor de RCHP en 1981, redefiniendo que la misión de la revista, en consonancia con la SOCHIPE, era "impulsar un Programa de Educación continuada en medicina infantil para pediatras y médicos generales" y "acentuar y perfeccionar su carácter de publicación científica, estimulando la producción de trabajos originales numerosos de gran calidad técnica, atractivos y de fácil lectura". Por primera vez, se profesionalizó la función del editor ${ }^{21}$. El año 1999 se agregaron las secciones "Preguntas al Experto" e "Imágenes Clínicas"22.

A fines del siglo XX la recepción de manuscritos en RCHP se vio amenazada por la competencia de las publicaciones extranjeras de alto impacto que captaban las publicaciones nacionale ${ }^{22,23}$. En una encuesta realizada a editores de revistas biomédicas chilenas, se refería que la mayor parte de los editores recibían la cantidad justa de manuscritos, optando por publicar pocos, pero buenos artículos, para mantener la calidad de las revistas ${ }^{24}$. En coincidencia, RHCP tuvo una baja en el número de manuscritos publicados.

Enfrentando los nuevos desafíos, el año 1998 la revista ingresó al mundo digital, a través de la publicación electrónica de libre acceso e integró una red latinoamericana de revistas a través de la red SciELO (Scientific Electronic Library on Line) ${ }^{25}$. Un año más tarde la SOCHIPE anunciaría la creación de una página WEB que alojaría a la revista ${ }^{26}$. En 2004, gracias a un ambicioso proyecto financiado por la SOCHIPE y con el apoyo de CONICYT, se logró la digitalización e incorporación a la base SciELO de la colección completa de los artículos publicados desde 1940.

En el año 1999 asumió el Dr. Francisco Cano como editor y la Dra. Francisca Ugarte como coeditora subdirectora; luego de cerca de 10 años, el cargo de coeditor fue asumido por el Dr. Paul Harris. En estas últimas 2 décadas, enfrentando los desafíos de la digitalización y globalización, RCHP se integró al grupo de Editores de Revistas de Sociedades de Pediatría del Cono Sur, formada por los equipos editoriales de las Revistas de las Sociedades de Pediatría de Argentina, Brasil, Bolivia, Paraguay, Uruguay y Chile ${ }^{27}$. Además, a partir del año 2005, comenzó a participar activamente en la Agrupación Chilena de Revistas Biomédicas. Ambas estrategias significaron importantes instancias de intercambio de experiencias y discusión de problemas comunes del trabajo editorial, llevando a la consolidación de la edición de las revistas de la región de acuerdo con normas internacionales.

En el año 2008, RCHP pasó de una gestión editorial manual basada en el correo electrónico, a una plataforma online basada en el Open Journal System (OJS) ${ }^{28}$. Esta modalidad de trabajo fue interrumpida por un período de 2 años (2015-2016) en que la revista fue editada por Elsevier, lo que constituyó un hito de importante aprendizaje e internacionalización, pero poco sustentable económicamente. En el año 2017 se retomó la plataforma OJS, pero esta vez no sólo en la interfaz de gestión editorial, sino que también en la publicación de contenidos, bajo la supervisión de expertos en informática biomédica y publicación científica, proceso que aportó significativamente en la profesionalización del trabajo editorial.

En el año 2014, en reconocimiento al trabajo de RCHP, esta fue re-incorporada a la National Library of Medicine (NLM). Al revisar los registros históricos, se puede apreciar que RCHP había sido indexada previamente en la plataforma, no obstante, debido a un cuestionamiento en la calidad de los manuscritos publicados y falta de evidencia de revisión por pares, fue eliminada en el año 1995, junto a muchas otras revistas latinoamericanas ${ }^{29}$. Con el objetivo de aumentar la 
presencia en el mundo académico, en los últimos años, RCHP fue presentada a Web of Science (WOS) de Clarivate Analitycs, y el 2017 fue incorporada al Emerging Sources Citation Index (ESCI) mientras se evalúa para recibir un Factor de Impacto (FI) de parte del Journal Citation Report (JCR).

Durante los años 2017 y 2018 se vivieron importantes sucesos en pro de fortalecer la excelencia y eficiencia de la revista, por ello se amplió el Comité Editorial con expertos nacionales de distintas disciplinas, además de un integrar un Comité Editorial Internacional; se incorporó la traducción al inglés de los artículos originales, se adquirió el identificador de objeto digital propio (DOI por su sigla en inglés), que corresponde a un código alfanumérico que identifica de forma única un contenido digital. Este avance permitió publicar anticipadamente los manuscritos venideros, con lo que se fue acortando significativamente el lapso de tiempos editoriales.

En la figura 1 se resumen los grandes hitos que ha vivido RCHP desde el ingreso al mundo digital y su impacto en términos de manuscritos publicados y citas recibidas.

\section{Bibliometría y cienciometría RCHP}

Durante los últimos años se ha experimentado un importante aumento en el número de manuscritos re- cibidos, que de 123 el 2012, aumentaron a 228 el 2016, y a 417 el 2019. En el primer filtro, realizado por el editor, de acuerdo con las normativas del año 1990, todo manuscrito que no cumpliera con los requisitos de publicación es "devuelto sin tramitar". Pero, en los últimos años aumentaron también los rechazos luego de revisión por pares, que pasaron del 10\% el 2012 al $54 \%$ el 2019 , reflejo de la alta calidad y flujo de manuscritos recibidos.

Como antecedente histórico, el proceso de revisión por pares fue implementado en el año $1980^{29}$. El año 1982 se recibieron 172 manuscritos, de los cuales 38 (22\%) "fueron rechazados, principalmente por defectos irreparables en su diseño o aspectos conceptuales" y 2 manuscritos fueron rechazados por "objeciones al diseño o contenidos del estudio" ${ }^{30}$. La primera vez que se publicó la "Nómina de árbitros" fue en 1995, en que se agradeció a los 100 revisores que entre los años 1992 y 1994, participaron arbitrando los manuscritos. Actualmente se extienden a más de 500 solicitudes anuales para hacer revisión de artículos, con una tasa de respuesta cercana al $50 \%{ }^{31}$.

Respecto al perfil de manuscritos publicados, en las primeras ediciones, la mayoría correspondían a revisiones, casos clínicos y actas de las sesiones del directorio de la SOCHIPE. A partir de los años 50, aumentaron los artículos de investigación u originales, y en los 60 la los comentarios en la sección "revista de revistas". En 1930 RCHP publicó 22 manuscritos originales, nú-

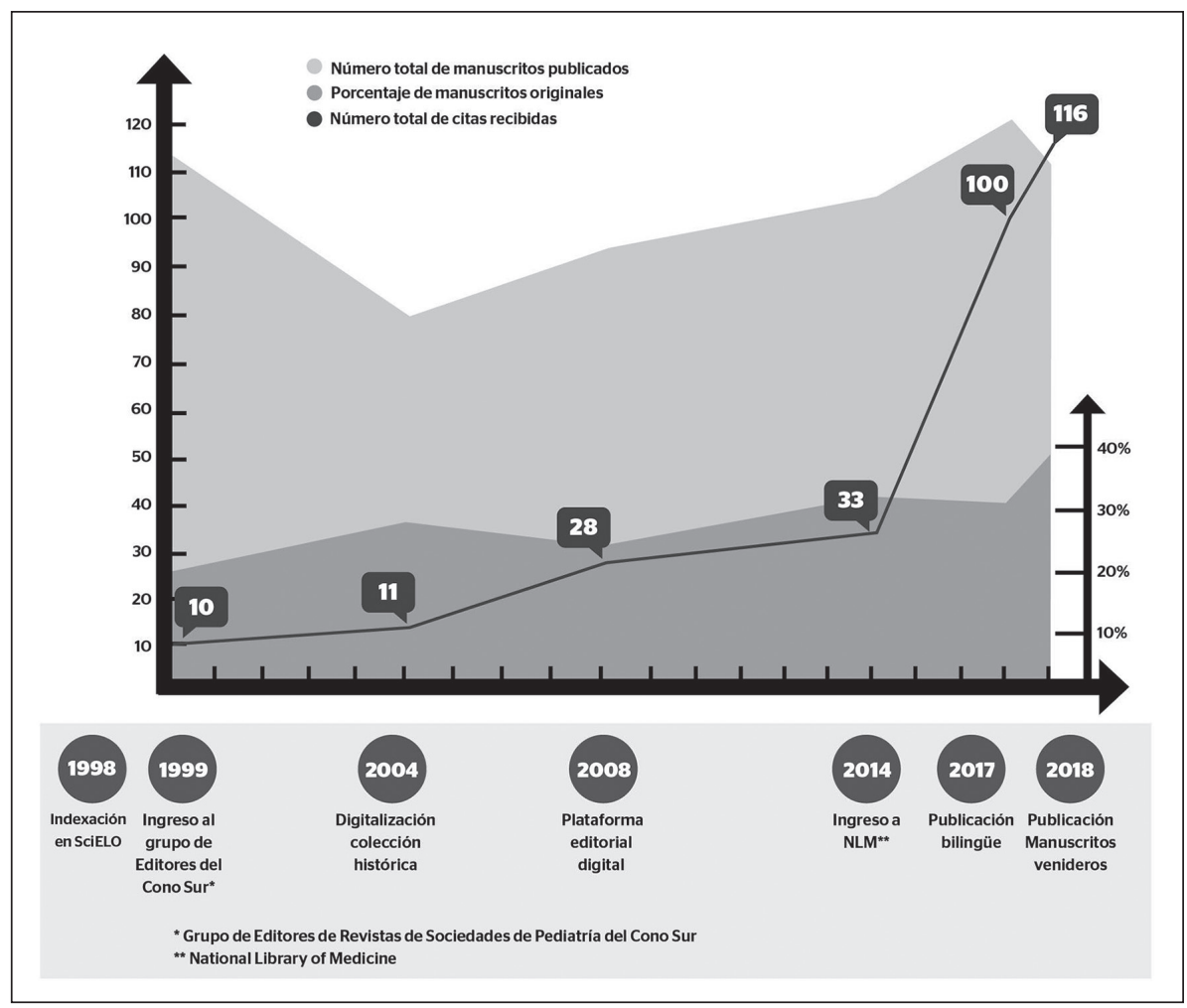

Figura 1. Hitos de Revista Chilena de Pediatría, desde su ingreso al mundo digital, e impacto en número de manuscritos publicados, porcentaje de manuscritos originales y total citas recibidas. 
mero que se mantuvo relativamente estable por 8 décadas, para comenzar a aumentar en forma progresiva en los últimos 10 años. A principios del siglo XXI menos del 30\% de los manuscritos publicados en RCHP eran originales, alcanzando en la actualidad el 40\% (Figura 1).

Todas las acciones descritas han ido consolidando a RCHP, que se ubica entre las seis revistas donde más publicaron los investigadores chilenos, en el período 2003-2013 32 . Durante la última década, la revista también ha sido elegida por investigadores de distintas partes del mundo, fundamentalmente Colombia, Argentina, Brasil y España, con un promedio anual de contribución de 20 manuscritos con autores extranjeros en los últimos 5 años. El año 2016, pasó de cuarto cuartil (Q4) al Q3 de ranking SJR Scimago, con un SJR de 0,2 y un $\mathrm{H}$ Index de $14^{33}$. Así es como en la actualidad, la RCHP se encuentra entre las 7 mejores Revistas Pediátricas Latinoamericanas, según el Ranking Scimago y CiteScor, gracias al significativo incremento en el número de citas por documento (figura 1) y el número de visitas a los manuscritos.

Recientemente RCHP se adjudicó un proyecto del concurso Fondo de Publicación de Revistas Científicas Chilenas, CONICYT, 2019, cuyo propósito es aumentar la visibilidad e incursionar en las redes sociales para mantener el liderazgo en los temas relacionados con la salud y bienestar de los niños de nuestro país.

\section{Conclusiones y desafíos para el Centenario}

Parafraseando las palabras del Dr. Humberto Garcés en 1960, para el aniversario de los 30 años de RCHP "el ideal que llevó a la creación de nuestra revista se ha cumplido ampliamente" ${ }^{\text {y }}$ estamos muy orgullosos de ello. Como revista representante de una sociedad científica, es fundamental mantener el equilibrio entre la sustentabilidad científica y la sustentabilidad económica, entre la promoción de la publicación nacional y la internacionalización, entre el impacto científico requerido para continuar ascendiendo en los rankings internacionales su rol en la educación continua, con impacto en la atención y cuidado de nuestros niños, en los distintos rincones del país. Todo esto no habría sido posible si no hubiésemos contado con el apoyo permanente de todas las directivas de la SOCHIPE.

\section{Conflicto de intereses}

Las autoras declaran no tener conflicto de intereses.

\section{Referencias}

1. Vargas N. Historia de la pediatría chilena: crónica de una alegría. Santiago de Chile: Editorial Universitaria; 2002.

2. Garcés H. 30 años de la Revista Chilena de Pediatría. Rev Chil Pediatr. 1960; 31(1):12.

3. Martínez G. Causas de la Gran Depresión de los años 30: aportes recientes. Revista Universitaria. 1983; 9:81-97.

4. Baeza A. La Revista de la Sociedad Chilena de Pediatría. Rev Chil Pediatr. 1930; $1(1): 3-5$.

5. Garcés H. Visión Retrospectiva de la Revista Chilena de Pediatría en su cincientenario. Rev Chil Pediatr. 1980; 51(1):3-7.

6. Cano F. La Revista Chilena de Pediatría en la realidad nacional. Rev Chil Pediatr. 2004; 75(2):105-6.

7. República de Chile, Dirección General de Estadística. Resultados del X Censo de Población de 1930. Santiago: Dirección General de Estadística; 1931.

8. Instituto Nacional de Estadísticas-Chile. Síntesis de Resultados Censo 2017. Santiago; 2018.

9. Pérez JC, Acuña Acosta EG. Mortalidad en Chile según las Tablas Abreviadas de Mortalidad por Sexo. País y Regiones
1919-2002. Santiago: Instituto Nacional de Estadística, Departamento de Demografía; 2004.

10. Instituto Nacional de Estadística. Estadísticas. [Online].; 2019 [visitado 31 12 2019]. Disponible en: https://www.ine. $\mathrm{cl} /$ estadisticas.

11. Allende S. La realidad médico-social chilena: (síntesis) Santiago; 1939.

12. Adriazola G. Experiencia en Planificación Familiar y Salud Pública en Chile. Rev Per Ginecol Obstet. 1980; 25(1):15-20.

13. MINSAL. Indicadores Básicos de Salud 2016. Santiago: Ministerio de Salud, Departamento de Estadísticas e Información de Salud, DEIS; 2018.

14. Zárate MS, González M. Planificación familiar en la Guerra Fría chilena: política sanitaria y cooperación internacional, 1960-1973. Historia Critica. 2015;(55):207-30.

15. Gutiérrez H. La Población de Chile: CICRED; 1975.

16. INE. Demografía y Vitales. [Online].; 2019 [visitado 3012 2019]. Disponible en: https://www.ine.cl/estadisticas/sociales/ demografia-y-vitales.

17. Milanich NB. Children of Fate: Childhood, Class, and the State in Chile, 1850-1930: Duke University Press Books; 2009.
18. Baeza A, Schonhaut L. Hospitalización del lactante. Rev Chil Pediatr. 2012; 83(3):290-4.

19. Palfrey JS, Tonniges TF, Green RJ. Introduction: addressing the millennial morbidity-the context of community pediatrics. Pediatrics 2005 ; 115(Supplement 3):1121-3.

20. Moraga F. La historia desconocida tras el logo de la Sociedad Chilena de Pediatría: Il Bambino de L'ospedale degli Innocenti. Rev Chil Pediatr. 2008; 79(2):190-8.

21. Toro C. Editorial. Rev Chil Pediatr. 1981(52).

22. Cano F, Ugarte F. Revista Chilena de Pediatría: Una invitación mirando al futuro. Rev Chil Pediatr. 1999;70(1):13.

23. Cano F, Ugarte F, Delucchi A, Sánchez I, García I. Publicaciones científicas: una identidad pediátrica nacional. Rev Chil Pediatr. 2000;71(3):181-2.

24. Oyarzún M. Cómo atraer más y mejores manuscritos para publicación en las revistas biomédicas chilenas. Rev Méd Chile. 2002;130(3):267-74.

25. Ugarte F, Schonhaut L, Harris P, Cano F. Revistas de Pediatría del Cono Sur: un camino de integración. Rev Chil Pediatr. 2012;84(5):481-3.

26. Cosoi E. Proyecto página web Sociedad 
Revista Chilena de Pediatría - L. Schonhaut B. et al

Chilena de Pediatría. Rev Chil Pediatr. 1999;70(6):509-11.

27. Schonhaut L, Cruces P, Cano F, et al. Vigésimo encuentro del Grupo de editores de las revistas de las sociedades de pediatría del Cono Sur. Rev Chil Pediatr. 2016;87(4):241-4.

28. Cano F, Ugarte. Revista Chilena de Pediatría 1999-2009: Logros de un
Período. Rev Chil Pediatr. 2010;81(1):911

29. Toro C. Proyecciones de la Revista Chilena de Pediatría. Rev Chil Pediatr. 1995; 66(5):235-7.

30. Toro C. Situación actual y horizontes en la publicación de trabajos en la Revista. Rev Chil Pediatr. 1983;54(3):303-4.

31. Schonhaut L, Millán T, Podestá L. Revisión por pares: evidencias y desafíos.
Rev Chil Pediatr. 2017;88(5):577-81.

32. CONICYT, Programa de Ciencia y Tecnología. Principales indicadores cienciométricos de la actividad científica chilena 2013. CONICYT; 2015.

33. SCImago. Revista Chilena de Pediatria. [Online]; 2020 [visitado 0301 2020]. Disponible en: https:// www.scimagojr.com/journalsearch. $\mathrm{php} ? \mathrm{q}=15786 \&$ tip $=$ sid $\&$ clean $=0$. 